\title{
Adding a Domestic Abuse Routine Prompt to the GUM Proforma: But Are we Asking the Question?
}

\author{
R Sacks, A Lavida, A Mears \\ Jefferiss Wing, St Mary's Hospital, London. Imperial College Healthcare NHS Trust
}

\begin{abstract}
Background
Previous studies have demonstrated high prevalence of domestic abuse (DA) within Genitourinary medicine (GUM) attendees with rates as high as $47 \%$ in female GUM attendees ${ }^{1}$ (nearly twice the rates seen in women in the general population), with $50 \%$ of men-who-have-sex-with-men and $66 \%$ of transgender having a lifetime experience of $\mathrm{DA}^{2}$.
\end{abstract}

The impact to the individual survivor their families and society is unquantifiable but in financial terms it is estimated that DA costs the UK approximately 15.7 billion per year' ${ }^{3}$. This has led NICE to conclude that 'the cost of domestic violence and abuse is so significant that even marginally effective interventions are cost effective' ${ }^{4}$. NICE explicitly recommend routine DA enquiry in all sexual health services ${ }^{4}$.

Studies have shown that despite patients accepting, and in fact expecting, routine DA enquiry ${ }^{5}$ when they attend healthcare services there may be reluctance on the part of healthcare professionals to implement this. Reasons suggested for this resistance include not wanting to pry, feeling that DA is a Social Care issue, time pressures and not knowing how to broach the subject or how to respond to and manage DA disclosures .

In July 2015, a routine DA prompt was introduced for all patients attending the Jefferiss Wing walk-in GUM clinic, St Mary's Hospital, London. DA guidelines, proforma and management flowchart were devised. On-going, tiered training was, and continues to be, provided at a basic level for all staff, and in-depth for Sexual Health Information Practitioners (SHIP) and for those volunteering to act as DA champions.

\section{Auditable outcomes:}

\section{DA question asked where safe (Target 100\%)}

(SAFE: quiet/confidential space, seen alone, no child $>18$ months present, professional interpreter if necessary)

2. Complete DA proforma if DA disclosed (100\%)

3. Offered SHIP referral for risk assessment if $\mathrm{DA}<3 / 12$ or on-going risk $(100 \%)$

4. Patient information leaflet offered if $\mathrm{DA}>3 / 12$ ago/no on-going risk (100\%) (In this context: 'On-going risk' may include a perpetrator due for prison release or returning to the area, children having witnessed DA/ children in the abusive relationship with (potential) ongoing contact with the perpetrator)

5. DA disclosures correctly coded (100\%)

An audit was conducted using the auditable outcomes listed above (see box) to assess if departmental DA guidelines were being followed.

${ }^{1}$ DV in a GUM setting-an anonymous prevalence study in women Loke W et al IJSA 2008;19: 747-51 ${ }^{2}$ Stonewall Gay and Bisexual Men's Health Survey 2012 Guasp A

${ }^{3}$ The Cost of Domestic Violence: Up-date 2009 Walby S

http://www.lancaster.ac.uk/fass/doc library/sociology/Cost of domestic violence update.doc (last accessed 25th June 2016)

${ }^{4}$ Domestic violence and abuse: multi-agency working. NICE Guidelines Feb 2014

${ }^{5}$ Women exposed to IPV: expectations and experiences when they encounter HCP: a meta-analysis of qualitative studies. Feder G et al. (2006) Archives of Internal Medicine 166: 22-37

\section{Method}

A retrospective case notes review was carried out on 100 consecutive, new, GUM patients aged over 18 years old, attending the walk-in service at the Jefferiss Wing, St Mary’s Hospital, London, from 1st October 2015.

\section{Results}

100 case notes were reviewed (59 female, 41 male).

1. $91 \%$ patients were asked about their lifetime experience of DA. Of the 9 patients that were not asked: $5 / 41(12.1 \%)$ male, $4 / 59$ (6.8\%) female. There was no reason documented explaining why the DA routine was not asked in any of these nine patients.

2. 5/91 (5\%) disclosed DA (all were female). The DA proforma was completed in $3 / 5(60 \%) .1$ of the 2 patients who did not have a proforma completed, declined further discussion and so was excluded from the analysis.

3. 1/4 (25\%) had current/on-going risk of DA, she was referred to the SHIP team for completion of a Domestic Abuse, Stalking and Harassment and Honour Based Violence risk assessment (DASH) and onward referrals where indicated.

4. In the remaining 3 patients, DA had occurred $>3 / 12$ ago and the patients were assessed as having no on-going risk. Of these three patients 1 accepted, 1 declined and 1 was not offered a patient information leaflet.

5. 2/5 (40\%) DA disclosures were coded correctly (using in-house codes).

\section{Conclusion}

This audit, conducted 3 months after introduction of a routine DA prompt for all walk-in GUM patients, demonstrated a high DA enquiry rate. Patients who were not asked were more likely to be male.

The departmental DA management guidelines were followed in the majority of cases where DA was disclosed (in terms of the DA proforma being completed and referrals to SHIP, where indicated).

There were low levels of accurate coding, using the new in-house DA codes.

\section{Limitations}

Small numbers of notes and the notes audited were consecutive, so a limited number of health care professionals work was reviewed

\section{Recommendations}

Audit presentation to the department, coding reminders to all staff and a coding prompt on the DA proforma

On-going regular DA training (both basic and in-depth)

Re-audit in 1 year 